\title{
Developing Nomad for Robotic Exploration of the Atacama Desert
}

\author{
David Wettergreen ${ }^{1, *}$, Deepak Bapna ${ }^{2}$, Mark Maimone ${ }^{2}$, Geb Thomas ${ }^{3}$ \\ 1. Intelligent Mechanisms Group, NASA Ames Research Center, Mountain View, CA 94035 USA \\ 2. The Robotics Institute, Carnegie Mellon University, Pittsburgh, PA 15213 USA \\ 3. Department of Industrial Engineering, The University of Iowa, Iowa City, Iowa 52242 USA \\ * Corresponding author: dsw@syseng.anu.edu.au, fax: +61-2-6279-8688
}

\begin{abstract}
Recent years have seen renewed attention on planetary exploration. Robotics is recognized as essential to upcoming missions of exploration. In this article we describe the ongoing efforts of the Nomad Project to develop robots for planetary and terrestrial exploration. The Nomad project is developing, demonstrating, and evaluating systems capable of long-distance, long-duration missions. In 1997 this work has resulted in the Atacama Desert Trek, in which Nomad, a mobile robot, explored the Atacama Desert of in northern Chile. Nomad's 45-day objective was to travel 200 kilometers across the rugged, barren landscape. We will describe the technologies for Nomad's transforming chassis, highdata rate communication, safeguarded teleoperation and autonomy, panoramic imaging and visualization, and remote science. We focus on issues of long-distance, long-duration exploration and describe the events and results of the Atacama Desert Trek.
\end{abstract}

Keywords: long-distance robotic exploration, navigational autonomy, panoramic imagery

\section{Introduction}

In recent years renewed attention has been focused on planetary exploration. In 1996 the Galileo probe entered the atmosphere of Jupiter [1] and in 1997 the Pathfinder lander bounced to a safe landing on Mars.[2] Mars Pathfinder carried a mobile robot, now well-known, named Sojourner. Mobile robots to explore our Moon, nearby asteroids, and to return to Mars are now in development.[3] Robotics is seen as essential to exploration of extraterrestrial bodies. The reasons are many and include safety, efficiency, durability, and expendability. To develop mobile robots qualified for planetary exploration, testing here on Earth under conditions and operations analogous to planetary missions will help to refine the necessary technologies and reveal the important issues. Harsh environments such as the deserts and polar regions of the Earth are accessible proving grounds for these experiments.

Nomad is a mobile robot designed for extended terrestrial and planetary exploration. (Figure 1.) In 1997, its mission was to travel 200 kilometers in 45 days across the rugged Ata- cama Desert of Northern Chile. This site provides close analogy to lunar and martian highlands. Nomad succeeded in this unprecedented traverse covering 223.5 kilometers of rough terrain while controlled autonomously or by operators thousands of kilometers away. This activity, called the Atacama Desert Trek, tested technologies critical to terrestrial and planetary field operations. It may prove to be a milestone in robotic exploration. [4]

The Nomad project seeks to develop, demonstrate, and evaluate robotic systems capable of long-distance, long-duration missions.[5] The ongoing project addresses issues of robotic configuration, communications, navigation, and guidance. We have also performed experiments to analyze and subsequently train scientists in using robots for exploration. In addition to advancing technologies for planetary exploration, Nomad's Atacama Desert Trek involved public participation in the robotic exploration. An immersive interface and safeguarded teleoperation enabled novice operators to safely drive Nomad from control centers in Pittsburgh, USA, Mountain View, USA, and Santiago, Chile.

This article is organized in two parts. We first 


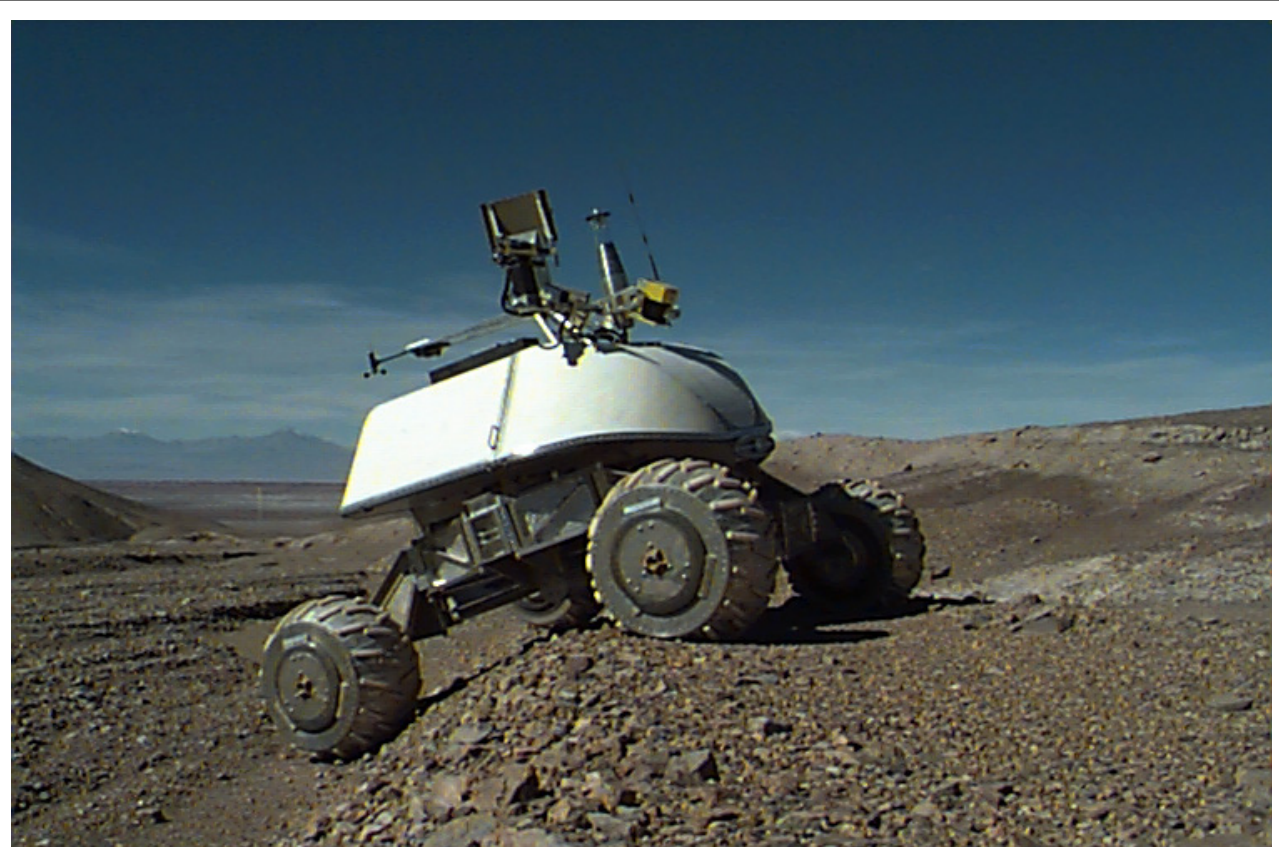

Fig. 1: Nomad in the Atacama Desert

detail the development of robotics technologies for Nomad's transforming chassis, high-data rate communication, safeguarded teleoperation, panoramic imaging and visualization, and remote science, in sections 2 through 6 respectively. These developments are previewed below and summarized in Table 1 . In the second part (sections 7 and 8) we focus on long-distance, long-duration exploration and describe the Atacama Desert Trek, the science experiments and public participation.

\section{Four-wheel drive and steering in a transform- ing chassis}

Nomad is designed with a unique chassis and suspension. It has four-wheel drive, four-wheel steering and a suspension that smooths the motion of the payload relative to the wheel excursions. The chassis can compact to less than $60 \%$ of its fully deployed area using the same actuators used for steering.

\section{High data-rate communication from a moving vehicle}

Communication with a moving vehicle is typically achieved with omnidirectional antennas. This limits the communication range, data rate, or both. To achieve high data-rates at long ranges, Nomad uses a high-gain, directional antenna. Using a specially designed pointing mechanism, we developed a technique to quickly aim the antenna in azimuth and elevation, to compensate for egomotion and to maintain alignment with the paired off-board antenna. This system can maintain communication at 1.5 Mbps.

\section{Safeguarded operation and obstacle avoidance in natural terrain}

Direct teleoperation requires a continuous low-latency communication link, as well as an attentive operator to identify the robot's path and avoid obstacles. Nomad reduces this dependence on a human operator using by onboard sensors, stereo cameras, and an onboard navigation computer to enable safeguarded teleoperation and autonomous driving. Nearby obstacles are modeled and mapped using stereo cameras, and registered using onboard position estimation. This allows Nomad to drive safely on its own, and to adjust automatically any operator commands that would cause it to drive into an obstacle.

\section{Immersive visualization and telepresence}

To monitor and guide its progress, and experience the environment that it explores, Nomad provides a comprehensive operator interface. Nomad carries a camera that generates pan- 


\begin{tabular}{|c|c|}
\hline Item & Details \\
\hline \multicolumn{2}{|r|}{ Physical } \\
\hline Mass & $725 \mathrm{~kg}$ \\
\hline Power & 3500W maximum \\
\hline Size & $\begin{array}{l}1.8 \mathrm{~m} \times 1.8 \mathrm{~m} \times 2.4 \mathrm{~m}, \text { stowed } \\
2.4 \mathrm{~m} \times 2.4 \mathrm{~m} \times 2.4 \mathrm{~m}, \text { deployed }\end{array}$ \\
\hline \multicolumn{2}{|r|}{ Locomotion } \\
\hline Wheel Size & $76.2 \mathrm{~cm}$ diameter $\times 50.8 \mathrm{~cm}$ width \\
\hline Static Stability & $+/-35^{\circ}$ \\
\hline Obstacle & $0.55 \mathrm{~m}$ height maximum \\
\hline Speed & $\begin{array}{l}0.5 \mathrm{~m} / \mathrm{s} \text { maximum } \\
0.3 \mathrm{~m} / \mathrm{s} \text { average }\end{array}$ \\
\hline \multicolumn{2}{|r|}{ Communication } \\
\hline Data Rate & 1.54Mbps maximum \\
\hline Equipment & $\begin{array}{l}\text { Wireless Ethernet bridge using high } \\
\text { gain antenna, Radio as backup }\end{array}$ \\
\hline \multicolumn{2}{|r|}{ Sensors } \\
\hline $\begin{array}{l}\text { Position } \\
\text { Estimation }\end{array}$ & GPS, gyrocompass, wheel encoders \\
\hline Navigation & Dual stereo wide-angle cameras \\
\hline \multicolumn{2}{|r|}{ Imaging } \\
\hline Panospheric & $1 \mathrm{k} \times 1 \mathrm{k}$ color at $6 \mathrm{~Hz}$ \\
\hline Compression & 60:1 maximum, wavelet algorithm \\
\hline \multicolumn{2}{|r|}{ Science } \\
\hline Climatology & Temperature, wind velocity, humidity \\
\hline Geology & $\begin{array}{l}2 \text { pairs of stereo cameras mounted on } \\
\text { a pan/tilt mechanism }\end{array}$ \\
\hline Mineralogy & $\begin{array}{l}\text { Eddy current sensor } \\
\text { Dual triaxial magnetometers }\end{array}$ \\
\hline \multicolumn{2}{|r|}{ Computing } \\
\hline Real Time & $\begin{array}{l}50 \mathrm{MHz} 68040 \& 40 \mathrm{MHz} 68030 \\
\text { running VxWorks }\end{array}$ \\
\hline Imaging & 200MHz Dual Pentium Pro running NT \\
\hline Navigation & 133MHz Pentium running Linux \\
\hline Science & 133MHz Pentium running Linux \\
\hline \multicolumn{2}{|r|}{ Operation Modes } \\
\hline Direct Teleop & $\begin{array}{l}\text { Remote driver, onboard safety } \\
\text { disabled }\end{array}$ \\
\hline $\begin{array}{l}\text { Safeguarded } \\
\text { Teleoperation } \\
\end{array}$ & $\begin{array}{l}\text { Remote driver, onboard safety } \\
\text { enabled }\end{array}$ \\
\hline Autonomous & No human intervention \\
\hline
\end{tabular}

Table 1: Nomad system specifications

oramic imagery in an ultrawide $\left(360^{\circ}\right.$-by- $\left.130^{\circ}\right)$ field of view. This camera provides a complete view of the surroundings in a truly immersive image. By combining panoramic visualization with easily comprehensible controls in a virtual dashboard we have created an interactive experience that we believe holds great promise for remotely-guided exploration.

At remote sites, live panoramic imagery is projected onto large screens to create a driving environment suitable to anyone. This provides people with a compelling experience and an opportunity to control a robotic explorer.

\section{Accurate scientific interpretation of remote environments}

Nomad carries sensors for remote geology and meteorite search. Its panoramic imagery allows scientists to efficiently localize Nomad and identify gross geology. Our experiments have shown the advantages of panoramic over traditional imaging for teleoperation and remote geology. High resolution imagery from science cameras mounted on Nomad enables characterization of rocks and features with accuracy never before achieved. A series of field experiments conducted during the Atacama Desert trek serve as a test for remote geological investigation, and validate new strategies for terrestrial and planetary exploration.

\section{Four-wheel drive and steering in a trans- forming chassis}

The first unmanned lunar rover, the Soviet Lunakhod, traversed $10 \mathrm{~km}$ of the Moon's surface.[6] Lunakhod, with a fixed wheel base and skid steering, was constructed with eight selfcontained electrically powered wheel modules. Later Russian designs for Marsokhod, a Mars rover prototype, have further developed the selfcontained wheel design.[7] Making a later lunar excursion, the American LRV had four-wheel Ackerman steering and was manually unfolded from stowed to deployed positions. Most recently, the Rocky series of micro-rovers, which led to the production of the Martian rover Sojourner, have used four-wheel explicit steering on a six wheel rocker-bogey suspension.[2] This suspension allows the rover to drive over relatively large discontinuities with smooth body motion.

For Nomad, the concepts of self-contained wheel modules and rocker-bogey suspension are combined with a new linkage design that pro- 
vides both explicit steering and an automatically-expanding wheelbase. Nomad features four-wheel drive, four-wheel steering on a unique transforming chassis. This propulsion, steering and suspension provide effective traction and mobility across loose sands, rocks and soils.[8] [9]

\subsection{Transforming chassis}

Nomad's chassis expands from a stowed to a deployed position with the same actuators used for steering. A pair of four-bar linkages collapse and expand to change the wheel base and steering direction. The chassis itself expands to 2.4by- 2.4 meters to improve stability and propulsion over variable terrain and collapses to 1.8by-1.8 meters for stowage, shown in Figure 2.
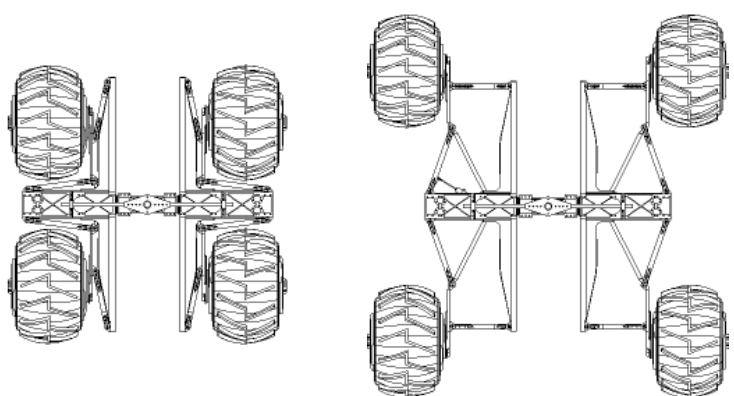

Fig. 2: Nomad chassis stowed (left) and deployed (right) showing steering linkages and pivot axis

Volumetric constraints motivated the unique design of the transforming chassis: constraints on the overall size of the robot (not to exceed the 1.8-by-1.8 meter dimensions of a launch payload), and the need to have sufficiently large wheels [10] to accommodate the incohesive layer of fine dust that covers lunar and planetary surfaces. Each of Nomad's wheels is a self contained system consisting of a tire, hubs, axle, motor and harmonic drive. All of the drive components are sealed inside the grouser-studded, aluminum tire.

\subsection{Multiple steering modes}

Nomad can steer in three modes: skid steering, point turning and double Ackerman. Skid steering is used only to position the robot for deployment and in the event of steering motors failure. Point turns are best used for large changes in orientation, for example when progress ahead is blocked. Shown in Figure 3,

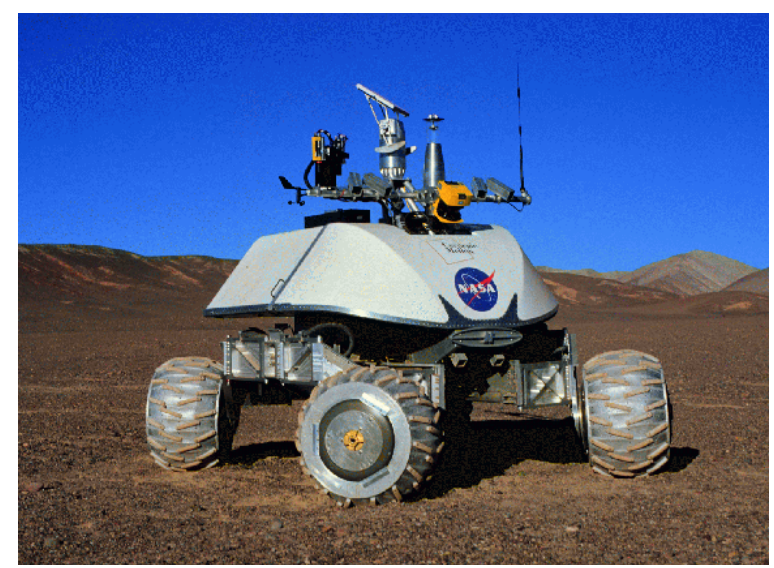

Fig. 3: Nomad with wheels positioned for turning in place the wheels must be steered to an extreme, $49^{\circ}$, before pivoting. The preferred mode of steering for Nomad is double Ackerman. Most automobiles have Ackerman steering on the front wheels; Nomad can steer its front and rear pairs. Just like a car, when turning, the wheels pivot by different amounts, because their distances to the center of turning are not the same. Nomad accomplishes this with two pairs of four-bar linkages - one pair on each side. A push-rod is attached to one axis of each linkage and is driven by two racks which are pulled in opposite directions by a single pinion placed between the two linkages. This pivots the front and rear wheel of each bogey by an equal but opposite amount.

\subsection{Averaging suspension using bogies}

The suspension averaging system acts to smooth the motions of the robot's body relative to the motions of its wheels. The two wheels on each side of the rover are attached through the steering linkage to a bogey which pitches relative to the body about a central axis. All wheels remain on the ground. (Figure 4.) With the pivot placed in the middle of each bogey, the vertical excursion of the pivot is the average vertical excursion of that bogey's two wheels. A similar averaging is experienced across the rover's body as center of the body lifts by an amount equal to the average vertical excursion of all four wheels. The pitch of the body is also the average of the pitches of the two bogies. This pitch, roll, and vertical averaging reduces the effects of rough terrain on the robot's body and its components. 


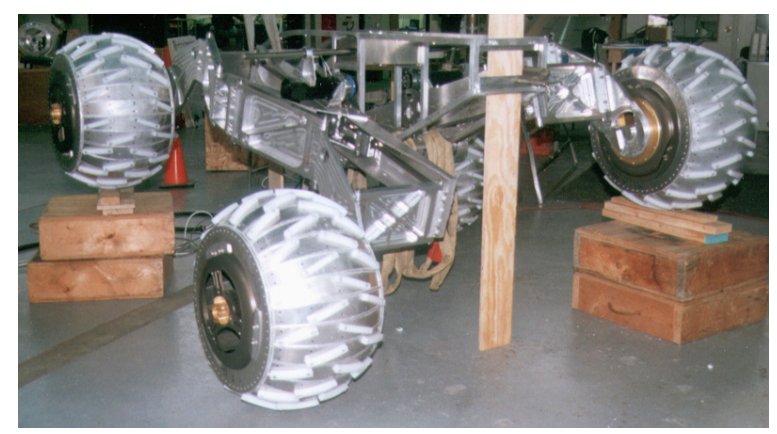

Fig. 4: Nomad chassis showing averaging effect of bogies

\subsection{Motion control by onboard computing}

Nomad's body shell encloses its computers, electronics, and sensors. A Motorola 68030 runs $150 \mathrm{~Hz}$ servo loops on all motion axes including the wheels and steering actuators, as well as sampling all digital and analog I/O like encoders and inertial sensors. This processor also calculates the correct steering actuator positions and wheel velocities to turn the robot along a given trajectory. The steering mechanisms on each side of the robot are independent, and must be positioned differently so that their turning arcs are concentric. The computer transforms turning radius commands into individual steering actuator and wheel servo references. This is accomplished by calculating the turning radius as a function of the actuator input, computing the appropriate wheel velocity, and using lookup tables to reverse the calculations. A Motorola 68040 handles the 8 port serial card. Devices attached to it are the gyrocompass, GPS, wireless modem, and other peripheral devices. This processor handles all communication, receiving commands and sending continuous telemetry.

Nomad is powered by a 5.0 kilowatt gasoline generator, not applicable to planetary exploration but necessary for this experimental system.

\subsection{Performance}

Several flaws have been revealed in the initial design and implementation of the chassis. Rocks occasionally wedge between the inner hub of the wheel and the wheel-link, causing severe abrasion and in some cases punctures to the hub and damage to bearing seals. This has been corrected by simple redesign of the wheel-link to eliminate the gap. A second problem was in the mounting of the actuator assembly within the wheel modules. Vibration caused the assembly to become misaligned, leading to gear wear and broken mounting bolts. It is now sufficiently secured against vibrations.

Nomad's wide wheel base provides the stable platform necessary for traversal of the rough terrain. It can descend slopes as steep as $38^{\circ}$, ascend slopes up to $22^{\circ}$, cross slopes at $33^{\circ}$ (roll), and surmount discrete obstacles up to $55 \mathrm{~cm}$ high. Nomad's maximum speed, limited by wheel motor velocity, is approximately $0.50 \mathrm{~m} / \mathrm{s}$.

\section{High data-rate communication from a moving vehicle}

Field robots commonly use omnidirectional antennas for communication with remote control stations. This restricts the bandwidth (nominally less than $100 \mathrm{kbps}$ ) and range (usually to less than $1 \mathrm{~km}$ ) due to the limited power available onboard the robot. [11] Instead, to achieve high data-rate communication over extended range, Nomad uses an directional, high-gain antenna. The challenge in this approach is to keep the directional antenna aligned despite the disturbances caused by driving over natural terrain. An active antenna-pointing mechanism is necessary to achieve this task.

\subsection{Communication scenario}

The ideal communication scenario for a mobile robot is to transmit directly to a satellite and from there to control stations [12]; however, the required size and mass of the antenna dish and transmitter equipment made this infeasible for Nomad. Another option is to transmit to a nearby relay station (located within 8-10 km) which then retransmits to a satellite. (Figure 5.) For a planetary mission the robot could relay through its lander, as Sojourner did. Because Nomad's telemetry requirements (1.0 Mbps, 10 $\mathrm{km}$ range) cannot be achieved using omnidirectional antennas, high gain directional antennas are required. Directional antennas on the robot and relay site must be aligned to receive transmissions and maintain a bi-directional link.

Nomad carries a wireless ethernet bridge and a radio for communicating with a repeater station. The wireless bridge, operating on the 2.4Ghz spread spectrum band, provides the high data rate link to transmit near real-time imagery 


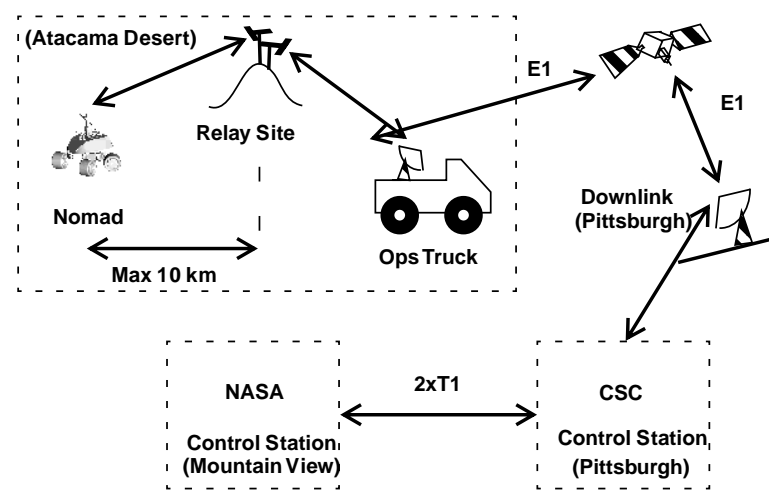

Fig. 5: Communications path from Nomad to Control Stations

via the high-gain antenna. A separate low-bandwidth radio system provides communication to allow the robot to recover from interruptions in the high-bandwidth link, in case line-of-sight between the antennas is obstructed or the pointing system suffers a tracking error. Operating in $900 \mathrm{Mhz}$ band, this radio uses an omnidirectional antenna and is less sensitive to the line-ofsight obstructions. Communication between the repeater station and a $1.8 \mathrm{~m} \mathrm{Ku}$-band satellite uplink station is achieved using a another wireless ethernet bridge. Information is uplinked to a satellite, where it is cross-strapped to a C-Band transponder and downlinked to ground stations.

\subsection{Antenna pointing mechanism}

The antenna pointing mechanism compensates for vehicle motion to keep the high-gain antenna fixated on the a relay station. Custom designed for Nomad, the antenna pointing device is a balanced mechanism (Figure 6) that can steer the antenna at high slew rates up to 60 degrees/second. It can rotate continuously in azimuth and adjust elevation from $-40^{\circ}$ to $+60^{\circ}$.

The pointing direction is controlled in an open-loop strategy; no direct feedback is received from the target as to whether the antenna is correctly aligned. This is because an appropriate error cannot be measured at the rate needed to correct for vehicle motion. The pointing error is generated based on the estimated location of the target, position and orientation of the robot, as well as the orientation of the pointing device with respect to the robot. Inverse kinematics give the required positions of the azimuth and elevation motors to compensate for vehicle motion and a PID controller drives the

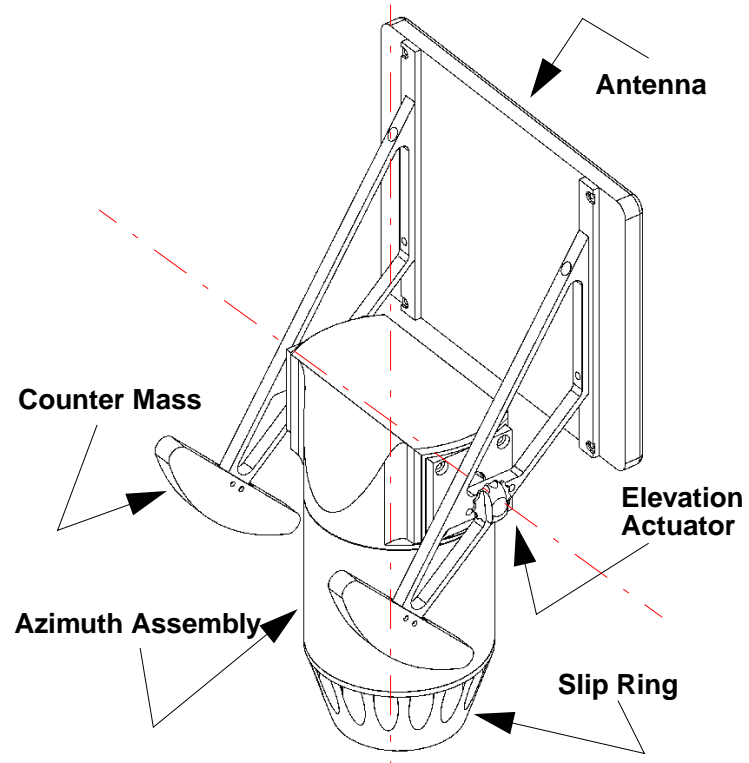

Fig. 6: Antenna pointing mechanism $\left(360^{\circ}\right.$ azimuth, $-40^{\circ}$ to $+60^{\circ}$ elevation)

antenna into proper position.

\subsection{Position estimation with inertial and GPS sensors}

To track the position of the repeater station located up to $10 \mathrm{~km}$ away, Nomad must accurately estimate its own position and orientation. This is accomplished by fusing data from different sources. A pair of GPS units configured in a differential mode enable resolution on the order of $20 \mathrm{~cm}$. Odometry from wheel encoder velocity data further refines position estimates. Orientation is provided by a gyrocompass/ inclinometer suite, which gives magnetic north heading as well as roll and pitch information to a resolution of $0.1^{\circ}$ and an accuracy of $1^{\circ}$.

Another method of localization, skyline navigation is being developed for Nomad. This technique takes a panoramic view of the horizon, and determines the rover's location by comparing the actual view to synthetic views generated from a digital elevation map (DEM) of the region being explored.[13] This technology was shown to successfully determine the robot's position to within 300 meters using a 1600 square kilometer DEM. It is not yet integrated with Nomad, however, so it has not contributed to the position estimates available to the antenna pointing system.

To achieve accurate pointing control, the nec- 
essary position estimates were generated using differential GPS, compass, inclinometers and encoder data. The expected cumulative error in pointing from all sensors is approximately $1.5^{\circ}$, which is well within the antenna's beam width of $2.7^{\circ} \cdot[14]$

\subsection{Performance}

Figure 7 shows the input data rate on the router recorded at random times for a period of about one hour. During this time, pointing

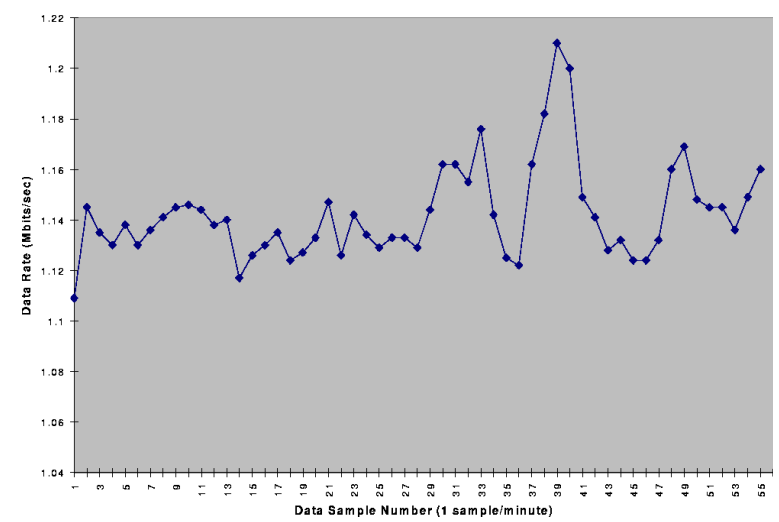

Fig. 7: Communications data rate per minute over a one hour

experiments were performed as the robot was commanded to move in circles on difficult terrains (obstacle fields) at different velocities.[15] As can be seen, even though the data rate fluctuated, it remained fairly high at all times. The communications latency is about 2.5 seconds due to the time-of-flight and short delays in various components.

Tracking error is introduced by several factors including the accuracy, resolution and bandwidth of each sensor, backlash in the drive train and time constant of the system. These errors are tolerable as the accuracy requirement is low due to the $2.7^{\circ}$ beamwidth of the antenna, hence open-loop control is sufficient. There is no means to measure the actual pointing angle (in a global reference frame), but this is not needed. If more accurate pointing is required, closed-loop control may be necessary, in which case feedback about the target direction is needed. This might be obtained by measuring the signal power strength, digital network bandwidth or packet latency.[14]

Nomad has communicated with relay stations up to $11 \mathrm{~km}$ away at data rates up to $1.5 \mathrm{Mbps}$. This is the first time this order of range and data rate has been achieved from a mobile robot. Communication dropouts do occur; they are primarily due to line-of-sight occlusions.

\section{Safeguarded operation and obstacle avoid- ance in natural terrain}

The vast distances and inherent communication delays encountered in planetary exploration present a fundamental technical barrier to direct teleoperation of planetary robots. The communication delay to the Moon (or the other side of Earth) is approximately 3 seconds-the delay to Mars can be more than 20 minutes. Twice that much time will pass before an operator can see the effects of their commands.

Nomad mitigates this limitation using onboard sensors and computing systems to autonomously distinguish between safe and dangerous routes. When these systems are enabled, Nomad will keep moving as long as it is safe, stopping only when it finds an obstacle it should not climb over and cannot avoid by simple steering. This strategy maximizes distance covered, and minimizes the number of required operator interactions.

\subsection{Navigation system}

Nomad's navigation system consisted of three primary components: position estimation, obstacle detection, and path planning. As described previously, position estimation was provided by differential GPS, gyrocompass, and inclinometer sensors.

Obstacle detection was provided by two pairs of rigidly mounted stereo cameras (Sony XC-77 with $3.6 \mathrm{~mm}$ wide-angle lenses) and the navigation computer (a 133Mhz Pentium PC running Linux). Nomad's stereo vision system had to have its field of view expanded, because Nomad was almost two and a half times the width of Ratler, the prototype on which the stereo vision system was first demonstrated.[16] This was accomplished using wide angle lenses and a new calibration procedure, for which we made a precisely machined, 6 foot tall calibration cube. The stereo vision sensors maintain their calibration, run successfully for up to nine hours each day, and have gone effectively untouched during 
more than 45 days of operation.

The Path Planning process proceeds as follows. A pair of images (e.g., something like Figure 16) taken by the navigation stereo cameras is processed into range data in less than one second. The stereo vision processing is explained in [17]. This range data is reprojected into an overhead view, yielding an elevation map of the area in front of the robot. Reprojecting in this manner allows us to reason about height obstacles directly in a world coordinate frame. Subsequent elevation maps are merged with the previous ones using the rover's new position estimate. Only a 20x20 square meter window of elevation data is kept onboard, since position uncertainties make it likely that data collected a few meters away is no longer properly registered with the rover's reference frame. The newly merged and truncated elevation map is then filtered to find all steep slopes, obstacles, dropoffs, and unknown areas (Figure 8). Having located all
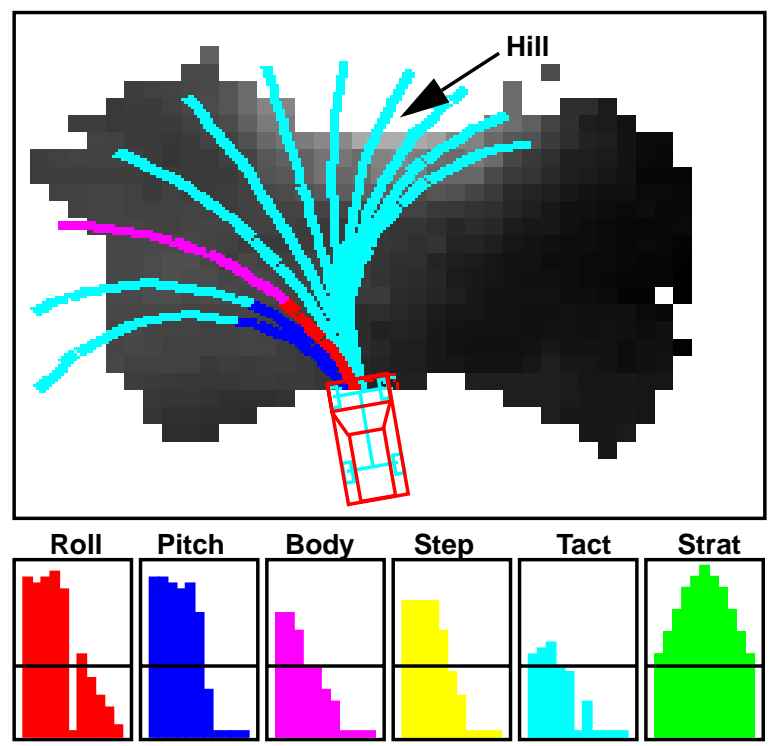

Fig. 8: Onboard obstacle detection for safeguarded teleoperation. Each potential turn angle is evaluated against roll, pitch, clearances, tactical and strategic measures.

potential hazards within a 10 meter radius, the planner considers all possible curved paths through the map as far as 10 meters ahead, and marks each one as safe or not-safe.

Three sources combine to determine the actual path traveled. First is the path planner's safety analysis, described above. Second is a preferred direction specified by a remote user.
Third, a point in the world (called a waypoint) may be specified as an eventual goal. These inputs are weighted and merged to find the optimal trajectory; see [18] for further details.

\subsection{Safeguarded driving modes}

Armed with the knowledge of path safety, Nomad controls its direction of travel using three different modes.

First, using Obstacle Avoidance mode, it drives randomly but safely. This was the mode of operation during the first third of the desert trek. At one point in the desert, Nomad came across an access road and chose it as the safest route, following it for nearly 400 meters.

Second, in Safeguarded Teleoperation mode, a remote driver provides a preferred steering direction at each time step; Nomad still chooses only safe directions, but prefers those near the user's direction.

Third, the final, most-used method is Waypoint Navigation. In this mode the user specifies a point in the world, meters or kilometers away. Nomad drives itself toward the specified point, avoiding obstacles along the way.

\subsection{Additional driving modes}

Several additional driving modes have been tested as well. Direct teleoperation is the most common mode, in which the remote user has direct control over the vehicle. In this mode the predictive safeguarding of the stereo cameras is turned off, so only internal safeguarding is enabled (e.g. tilt sensors, real time computer consistency checks).

Two types of patterned search modes are available: uniform grid and arbitrary waypoints. Both controlled the steering direction automatically by using the internal position estimation sensors to plot a course.

In the uniform grid mode Nomad is dynamically servoed (typically at $10 \mathrm{~Hz}$ ) as it executes a search of a rectangular area. Nomad drives back and forth in evenly spaced rows, completely covering the search area along the way. This type of control provides the capability to deploy a sensor and exhaustively search an area, a technique critical to future terrestrial surveys for meteorites.

The waypoint mode of patterned searching is similar to the waypoint navigation mode 
described above, but allows the vehicle to operate without any stereo vision data. In this mode Nomad visits an ordered list of global coordinates. All processing and control are performed onboard the vehicle, with the lists of goal points provided by the remote operator. Once Nomad reaches a goal location, it immediately starts driving toward the next one.

\subsection{Performance}

During the Atacama Desert Trek, Nomad always chose a safe path and found obstacles on its own. Unfortunately, the software model of the novel steering mechanism's kinematics was not complete, and the turn-in-place maneuver that had been an integral part of obstacle avoidance behavior in Ratler were not always available. So, although Nomad successfully found obstacles and stopped in front of them, sometimes a human operator had to back it away from the obstacle.

Finally, a network communication bug prevented the safeguarded teleoperation mode from being exercised more regularly. The bug caused an unreasonable delay in achieving a link between the remote user interface and the onboard safeguarding system; start-up time varied from a few seconds to more than 20 minutes. This odd behavior was not manifest during USbased trials, only over the live satellite connection, but it imposed an unreasonable delay in nominal operations and therefore safeguarded teleoperation was used infrequently.

The primary performance metric for the desert trek was the total distance traveled during the 45 days of field operations: 223.5 kilometers. Direct teleoperation contributed over 130 kilometers to the total distance traveled, and patterned searches contributed 63 kilometers: of these, uniform grid mode accounts for 57 kilometers, and waypoint mode 6 kilometers of travel.

The safeguarding system was on call throughout the mission, and was typically run ondemand as circumstances warranted, such as during lunch breaks or loss of communications. The graph in Figure 9 illustrates the daily total distance traversed in the various modes. In summary, Nomad was driven for a total of 21 kilometers of autonomous driving $(1.7 \mathrm{~km}$ obstacle avoidance, $19.3 \mathrm{~km}$ waypoint navigation), and 7

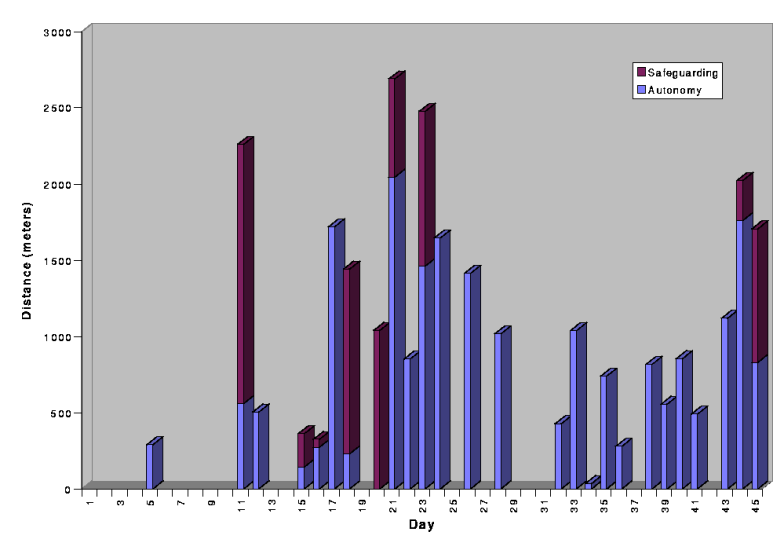

Fig. 9: Distance traveled per day in autonomous or safeguarded modes during the Atacama Desert Trek.

kilometers of safeguarded user-driven teleoperation, at speeds up to $43 \mathrm{~cm} / \mathrm{second}$.

\section{Immersive visualization and telepresence}

The Nomad interface combines panoramic visualization with easily comprehensible telemetry display and operator commands, a combination that is distinct among interface designs. In most other systems, the operator examines the images taken by the robot, decides where to direct the robot, and executes the directions through a joystick or some other input device.[19][20] Other researchers have directed their robots based on a model of the remote scene [21] or have integrated both the modeling and immediate image approaches into a single system. [22][23] Nomad's interface is unique in that it uses three-dimensional modeling to assist in the visualization of the remote scene, employs live panoramic imagery, and uses a sophisticated, yet comprehensible interface to send commands to the robot. This combination of technologies creates an interface that provides a compelling sense of presence and is readily usable by expert and novice alike.

\subsection{Panospheric imaging of complete surround- ings}

Whereas traditional cameras provide limited field-of-view of perhaps 35-60 degrees, Nomad carries a panospheric camera that generates imagery with an ultrawide field of view, 360 by 130 degrees; as shown in Figure 10.[24]

The camera captures a $360^{\circ}$ view of the surrounding terrain from straight down to $40^{\circ} \mathrm{s}$ 


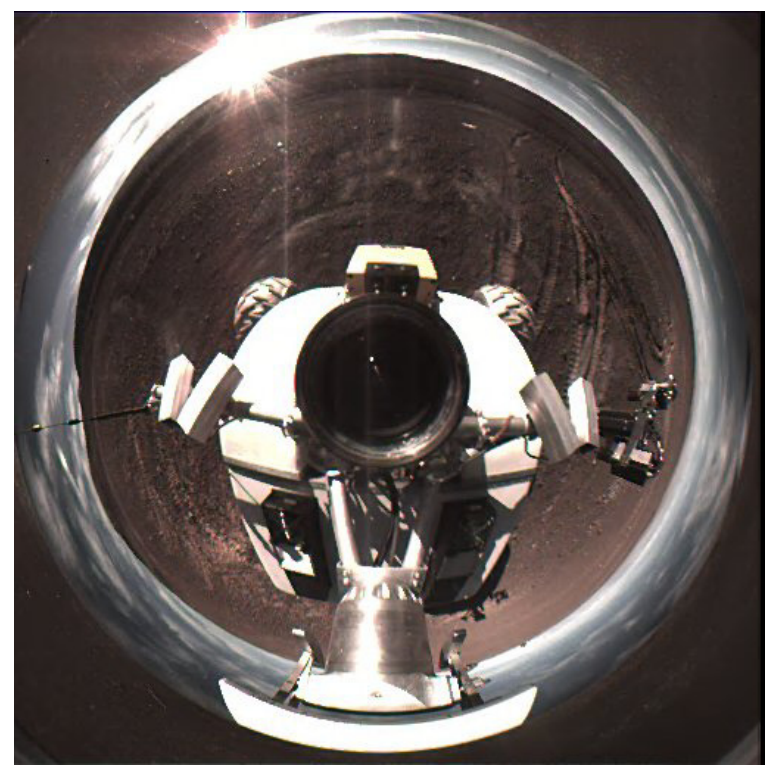

Fig. 10: Image from the Panospheric Camera

above horizontal using a spherical mirror mounted above a vertically oriented digital camera. The mirror is a $70 \mathrm{~mm}$ plano-convex lens with a $60 \mathrm{~mm}$ diameter chord. The scene is imaged with a $1024 \times 1024$ color CCD chip with an alternating color mask for an effective color resolution of $512 \times 512$. Images are read out in vertical columns at up to $6 \mathrm{~Hz}$ and shifted from the digital camera via a high-speed frame grabber to a dual-processor $200 \mathrm{MHz}$ computer for real-time image compression.

\subsection{Transmitting panospheric images}

We have developed a method to take an image, compress it, decompose it into packets, transmit these to multiple remote computers, reconstruct and finally, uncompress the original image.

A wavelet-based compression algorithm [25] reduces images from 786 kilobytes $(512 \times 512 \times 24$ bits $)$ to about $30-60$ kilobytes, using a compression of 15-30:1. One processor compresses images at a maximum of $2.5 \mathrm{~Hz}$. The compression processor queues images into shared memory where a messaging processor breaks each image into 8 kilobyte packets.[26] These packets are multicast from Nomad and received in multiple locations. Each packet contains a packet identification code, the total number of packets in the image, and a variable length data block. This queuing scheme decou- ples image capture and compression from the network delay.

As each remote site, multiprocessor computers reassemble images from the packets. Complete but still compressed images are passed through shared memory to be uncompressed and displayed. More than one million panospheric images were transmitted during the desert trek.

\subsection{Dewarping panospheric images}

The panospheric camera generates highly distorted images. To present images that are less difficult to interpret, the interface dewarps the images by rendering them as surface textures on a curved surface. The shape of the surface is tailored to the optical properties of the camera. To construct it, a ray is traced from each pixel in the image plane through the reflection on the mirror surface and then for a fixed distance into free space. Joining the ends of these rays forms the surface mesh. Each vertex in the surface mesh is associated with a image coordinate. A perspective viewpoint at the center of the mesh provides optically undistorted images rendered to the screen. As new images arrive from Nomad, they are mapped onto the mesh, but do not affect the surface's geometry. (Figure 11.) This technique can efficiently employ texture mapping hardware to dewarp the images.

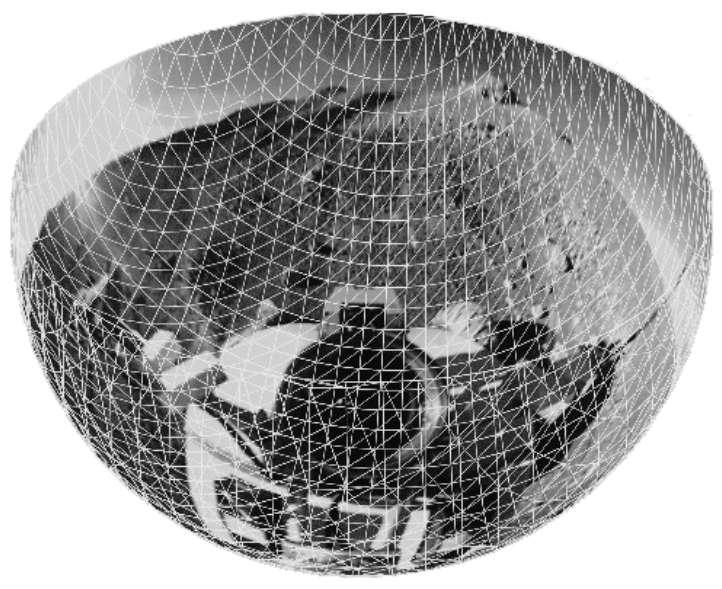

Fig. 11: Panospheric image textured onto dewarping surface

Several of other researchers have also investigated panoramic imaging using a variety of imaging and dewarping techniques [27][28], typically for real-time video teleconferencing.[29] Most approaches differ from ours in 
that the imagery is typically of lower resolution but higher bandwidth. Typically the effective lens must have a single center of projection, but this is not necessary with Nomad's texture mapping method of dewarping.

\subsection{Telepresence Interface}

The Telepresence Interface uses the encompassing imagery from Nomad's panospheric camera to create a sense of presence in the robot's environment. Operators can look around, from Nomad's perspective, with smooth and natural motions, with the screen refreshed at $30 \mathrm{~Hz}$, and with new images appearing seemlessly. (Figure 12.) By changing the orientation of the

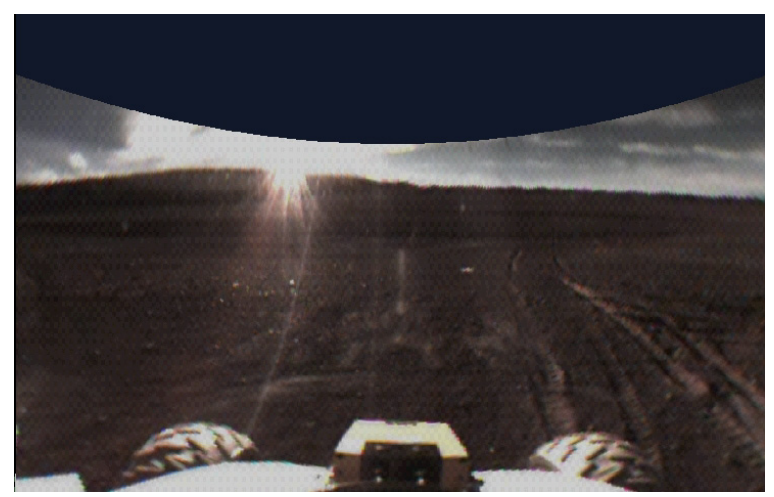

Fig. 12: Display of panospheric imagery (from Figure 10)

rendering viewpoint with a mouse, the viewer can smoothly spin their viewpoint inside the textured sphere to view Nomad's environment in any direction.

The target update rate for panoramic imagery was $7.5 \mathrm{~Hz}$ however, to date, the average is approximately 1 image per second ( 2 maximum) with a latency of approximately 5 seconds. The rate is limited by the speed that image packets can be queued and transmitted across the network. The latency is introduced by the time required to compress and decompress each image. Since the operator's interaction with each image $(30 \mathrm{~Hz})$ has a much faster refresh rate and smaller latency than overall the image acquisition process $(1 \mathrm{~Hz})$, the Telepresence Interface still imparts a feeling of real-time control; the effect of teleoperation latency on the experience is minimized.

\subsection{Virtual dashboard for both novice and expert operators}

To guide Nomad and monitor its progress we created the Virtual Dashboard, shown in Figure 13.[30] The Virtual Dashboard is constructed of interchangeable display panels each of which provides a representation of aspects of Nomad's state in graphical and numeric formats. The robot's position is plotted on aerial images, and the pose is rendered in 3-D with everything updated in real-time. An operator can quickly assess Nomad's condition and, using a mouse, command the robot.

A telemetry panel displays position, pose and state information for the operator. (Figure 13, bottom left.) Several numeric displays show Nomad's position in world coordinates, the pitch and roll angles, latitude and longitude, chassis bogey angles and turn radius. The telemetry panel contains a message window in which activity notices and warnings about approaching or exceeded preset limits, like vehicle roll angle, are displayed (along with an audible alarm). With the telemetry panel menus, the operator can deploy or stow the wheels, change motor servo parameters, reset the trip counter, make $\log$ entries, or vary the telemetry rate.

The aerial tracking panel (Figure 13, top right.) retrieves aerial images from a database and overlays an indication of Nomad's position in the terrain. The operator can create bearing and waypoint commands using mouse clicks and drags within the aerial image. Each aerial image comes with a set of equations that relates the pixel location within the image to global coordinates. The output of Nomad's position estimator localizes it within the image. As it moves, the dashboard puts down a markers so that the rover's path is traced. The usefulness of this dashboard panel depends on both the accuracy of the robot's position estimation and the accuracy with which the aerial images are recorded. We discovered that the position, and importantly the orientation, of many of our aerial images varied and in some instances caused errors of up to 100 meter in plotting markers.

The science camera panel displays rover compass data and contains the controls for the science imaging package on Nomad. (Figure 13, bottom, second from left.) The compass rotates 


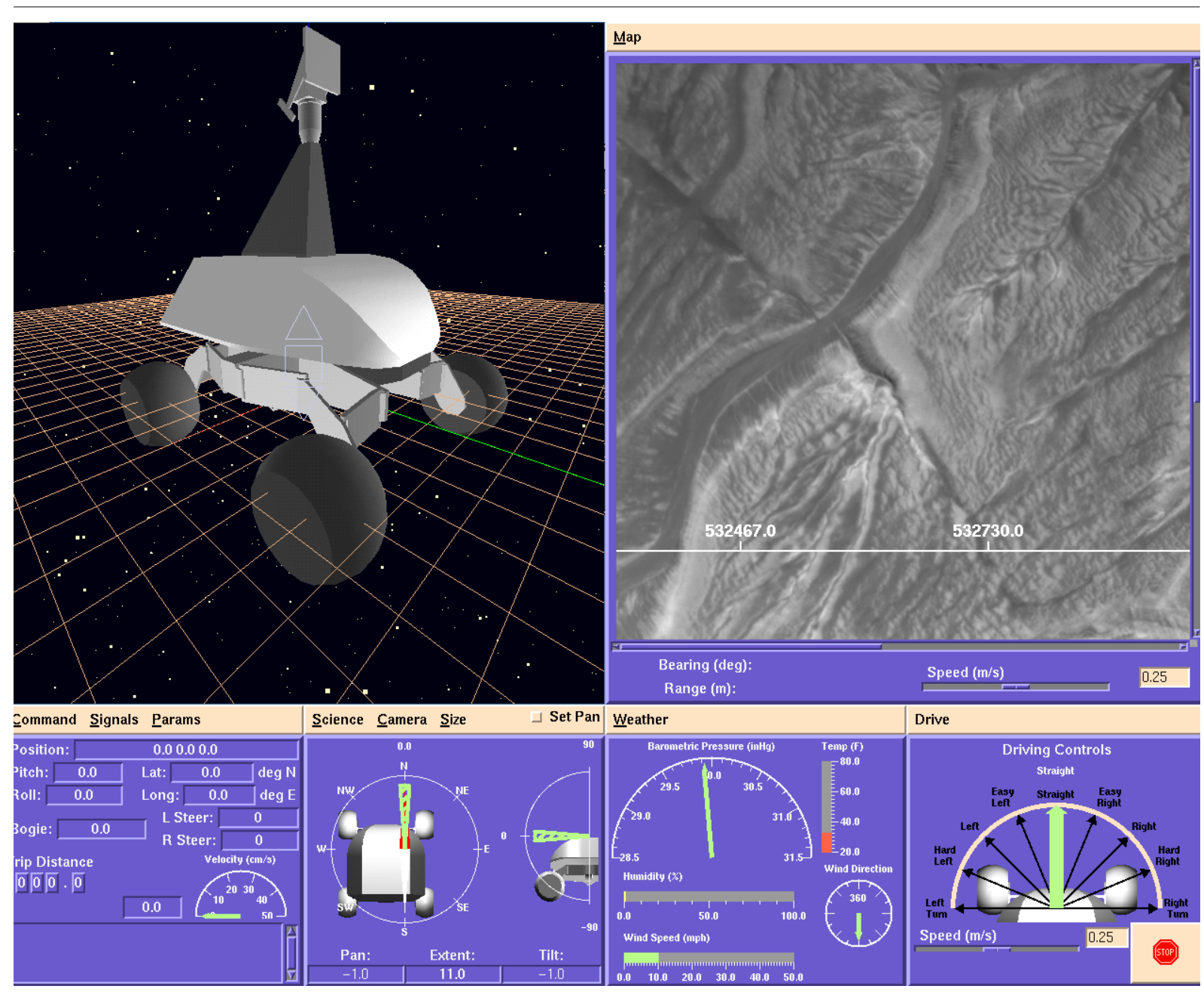

Fig. 13: Virtual Dashboard

to reflect the incoming telemetry. The camera controls allow the operator to maneuver the pantilt device using the mouse. Typically, scientists want images from an absolute bearings. By overlaying the pan controls on top of the compass display, there is no need to translate from absolute directions to relative pan-angles. This simple detail improves efficiency and reduces errors.

The driving control panel contains the controls for sending direct teleoperation commands to the rover. The operator sets the turn radius by rotating a radial dial and sets the speed and distance controls with slider bars (or keyboard input). (Figure 13, bottom right.) Once all the parameters of the drive command have been set, the user simply clicks to send the command. An emergency stop button immediately sends a low- level command to shut-down motor servos.

The Virtual Dashboard incorporates a rendered 3-D model of Nomad with each articulation updated at 5Hz. (Figure 13, top left.) This provides an accurate representation of Nomad's relative position, orientation, and kinematics, which can be viewed from any perspective. This virtual environment model was a central component of previous rover interfaces [32] but, interestingly, because Nomad does not currently transmit a detailed local terrain model there is insufficient context to interpret the scene. Many operators choose not to view the virtual Nomad because they find it misleading without more complete information about its relationship to the surrounding terrain. 


\subsection{Performance}

During the Atacama Desert Trek, experienced drivers at NASA viewed the Telepresence Interface on a large projection screen while sitting in front of a workstation running the Virtual Dashboard in an arrangement similar to the windshield and dashboard in a car. Nomad's has been teleoperated along detailed paths designated by a team of planetary scientists.

At most other times, Nomad was driven by novice drivers, often a member of the public. These operators drove anywhere they wished and viewed panoramic imagery in a theater with three projectors illuminating a $4 \mathrm{~m}-\mathrm{by}-12 \mathrm{~m}$ $\left(220^{\circ}\right)$ curved screen. Most of the 100 novice drivers found the controls easy to learn, and after a few minutes of explanation, were able to drive Nomad independently. Novice drivers tend to use the visual information from the panoramic image as their primary source of driving feedback, only occasionally glancing at the dashboard for roll and pitch information. Because of their greater dependence on the panoramic display, which carried with it a 5 second latency, novice drivers also tended to over-steer the vehicle when driving at top speeds. Experienced drivers made greater use of the compass, position, and pitch and roll displays.

In all, the Nomad's operator interfaces performed well for two types of driving situations: experienced drivers with specific mission objectives, and novice drivers exploring at random. Learning time was short, on the order of minutes, for drivers.

\section{Accurate scientific interpretation of remote environments}

Tools that facilitate imaging and analysis are essential for remote scientific investigation. Nomad incorporates instruments for experiments in telescience, specifically for geological investigation. Along with its panospheric camera, it carries stereo color cameras mounted on a pan-tilt device and an eddy current sensor (metal detector) and two 3-axis magnetometers to find ferrous materials.

\subsection{Imaging camera and pointing device}

To determine if poor imaging resolution limited scientific accuracy in previous field experi- ments [31][32], the science imaging system for the Nomad field experiment was designed to match the resolution of the foveal region of the human eye, about 0.25-0.33 milliradians per pixel.

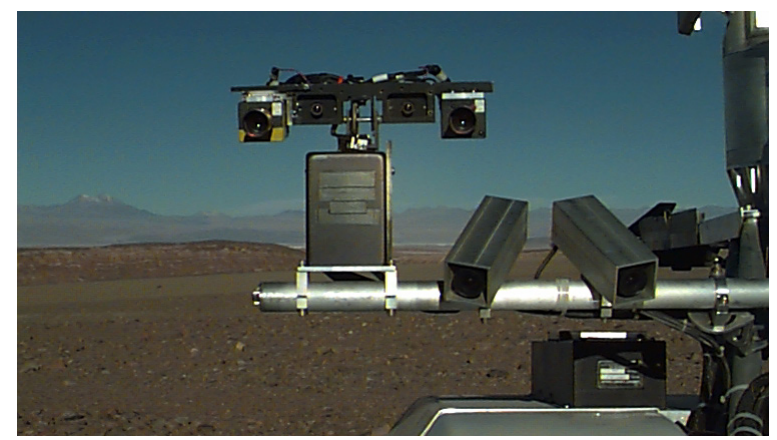

Fig. 14: High-resolution color stereo cameras at $25 \mathrm{~cm}$ baseline (outer pair) and low-resolution cameras at $12.5 \mathrm{~cm}$ baseline (inner pair) on a pan/tilt device. Also visible are the right side navigational stereo cameras.

Triple-CCD cameras, Sony XC-003's, with 1/ 3 inch imagers and C-mount lenses, were selected and with a $25 \mathrm{~mm}$ lens, provide 0.30 milliradian per pixel image resolution. This results in an $11^{\circ}$ field-of-view. To extend the field-of-view, mosaics of multiple images may be taken and then assembled into a larger image. Monochrome cameras provide a wider field-ofview $\left(33^{\circ}\right)$ for sighting the high resolution cameras. (Figure 14.) The color cameras have a $25 \mathrm{~cm}$ baseline and are verged and focused at 5 meters. The cameras are mounted 1.5 meters above the ground. These settings yield the best resolution for objects on the ground about 3 meters in front of the robot. (Figure 15.)
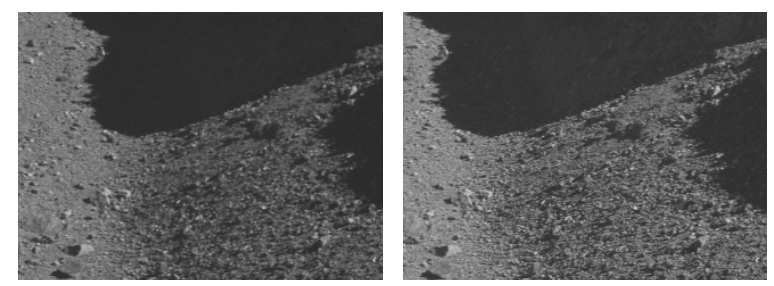

Fig. 15: High-resolution stereo image pair of dry streambed.

The science imaging system is designed to be easy to integrate onto Nomad. It consists of a pan-tilt device, the two high-resolution color cameras, and two monochrome cameras. An adjustable mounting bracket allows the two color cameras to be precisely aligned in pitch, roll, and vergence and locked down to maintain 
alignment even under prolonged vibration. An electronics box containing the computer, power supplies, and control electronics mounts inside Nomad's body shell.

\subsection{Modeling from science images}

Photorealistic terrain models allow scientists and engineers to learn more, and more quickly, about the remote location by allowing them to virtually explore the terrain. The science imaging system produce stereo pairs that can be used to build models of the surroundings. (Figure 16.)

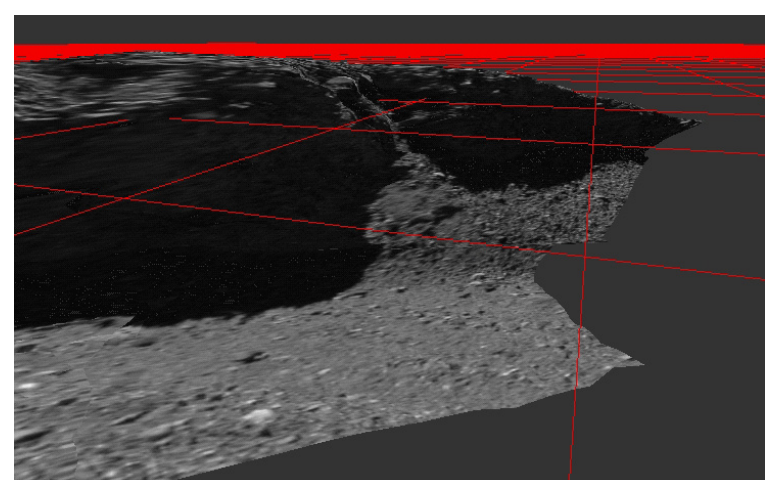

Fig. 16: Terrain model built from 12 stereo pairs (including Figure 15) with images overlaid and viewed orthogonally.to the direction of observation.

These terrain models help scientists define morphology of rocks, outcrops, washes and other surface features. They are also useful for determining site trafficability. A plan view of the rover surroundings can be used to determine feature distribution and alignment, to estimate distances, and to plan paths.[33]

\subsection{Reliable communication of science images}

High-resolution images for scientific analysis are required infrequently and need reliable transmission when they occur. However, loss of communication to the robot explorer can last for seconds or even minutes, so a scheme was developed to provide fast and (selectively) reliable transmission of data through unreliable communication links.

A connectionless UDP scheme was chosen because it avoids acknowledgment delays, important for streaming panospheric images. However since it provides no guarantee that packets will be delivered, an algorithm was designed to send packets out at a specified data rate and then request missing packets as they are detected. A sequence packet contains identification codes for all the packets that make up a complete science image. Whenever a gap in the sequence is detected, the missing packets are requested by the receiver. Each image packet also references the image sequence it corresponds to, so if the sequence packet has been lost, a request is sent for it too. In order to transmit mosaics, or groups of images, the scheme must cope with many partial images in transit simultaneously. After a long communication interruption, when the link is reestablished all available bandwidth is used to resend missed image packets. Due to the variable latencies in radio and satellite networks, it is difficult to analyze or predict changes in available bandwidth. To avoid bandwidth saturation and additional packet loss, the time since the transmission buffer last cleared is used to adjust the transmission rate. The timing and scale factors must be determined empirically but recovery from link losses of up to several minutes is routine.

This multi-destination, reliable data transmission performs well under harsh, real world conditions. The science package has transferred over 400 megabytes of images over heavilyloaded radio, satellite, wide, and local area links. This protocol could be used with multi-cast UDP packets to further improve performance when supplying a large number of receivers although the retransmission of dropped packets might require proxies to minimize traffic with the robot.

\section{Long-distance, long-duration robotic exploration}

\subsection{Atacama Desert}

Located in northern Chile, the Atacama Desert (Figure 17) proved to be an ideal setting for testing and demonstrating robotic capabilities for planetary exploration. The Atacama includes a variety of features and characteristics that make it a unique place to perform experiments on planetary-analog surfaces: craters from meteoritic impacts, volcanoes, rocks and sand fields, total lack of vegetation due to the absence of rain $(1 \mathrm{~cm} /$ year from fog), and ancient-and now dry-lake beds and sea floor. Its heavily eroded topography, rocky terrain and loose 


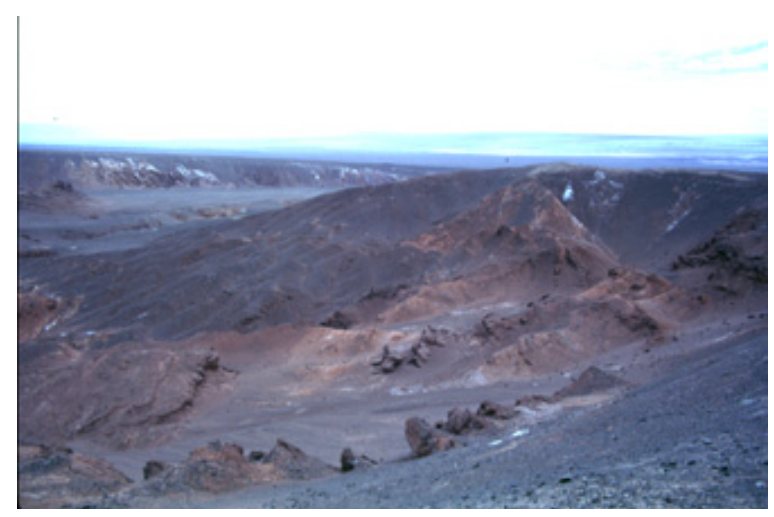

Fig. 17: Terrain in the Atacama Desert of Chile

sands combine to create a landscape similar to that found on areas of the Moon, and Mars.

The selected site near $23^{\circ} 20^{\prime} \mathrm{N}, 68^{\circ} 37^{\prime} \mathrm{W}$, is a mountain range called Domeyko just west of the Salar de Atacama, and is considered to be the most rugged part of the desert. The average elevation in the area of the trek is $2400 \mathrm{~m}$; the diurnal temperature variation is from $0^{\circ}$ to $25^{\circ} \mathrm{C}$. This site provided varied topography suitable for antenna placement, views of the surrounding landscape, and operational access. The Atacama's location within the same time zone as eastern United States also simplified coordination of operations.

\subsection{Synopsis of the desert trek}

The objectives of the Atacama Desert Trek included multiple technology evaluations and demonstrations, remote science, and public outreach. Accomplishing all these in a month and a half required much planning and physical effort. Following is a synopsis of the major events.

Nomad arrived in the desert on June 9th, 1997 , just 8 days before the trek was to commence. The robot was assembled and tested, the communication link was established (June 15th) and debugged, and the relay station and operations truck were configured before Nomad's public debut on June 18th (Day 1). Of course, we wanted to make sure everything was working so we started the trek the day before, on June 17th. The mileage tick started on Day 0 when the robot was operated from the NASA Ames Research Center. On its first day of the mission, Nomad traversed approximately $1 \mathrm{~km}$ (or $0.5 \%$ ) of its objective distance.

On Day 1, the robot was driven by the opera- tors at both the Carnegie Science Center and NASA. The public debut of Nomad had hundreds of visitors watching Nomad traverse the harsh, but beautiful terrain. Initial progress was slow with Nomad traveling less than 1 kilometer on several days.

The first autonomous operations were demonstrated on Day 5 when Nomad traversed autonomously for 290 meters. Day 6 through Day 10 of the mission were dedicated to the remote science operations, discussed in detail in the next section.

A mechanical failure occurred on Day 12. The motor in the right front wheel came loose from its mounting. This, however, provided an opportunity to test Nomad's abilities as a 3wheel drive; it worked. Day 13 was another milestone as the first novice driver took charge of the robot. From this point, almost every day novice drivers operated Nomad. Base camp was relocated by about 15 kilometers on day 17 .

On Day 22, Nomad traversed $8.6 \mathrm{~km}$, covering total of $52.6 \mathrm{~km}$ so far. It took $50 \%$ of the mission time to achieve first $25 \%$ of the traverse. On Day 25, Nomad established another milestone in remote traverse by successfully operating at night. From then on it was operated almost every night, performing patterned searches of predetermined areas. The next day, Nomad covered a record $26.8 \mathrm{~km}, 64 \%$ of it being traversed at the night. At the end of this day (Day 26), the cumulative distance was $105.3 \mathrm{~km}$. There were 18 days left to cover the remaining distance. During the Day 23-26 period, Nomad covered more than $50 \mathrm{~km}$.

The skyline navigation tests were started Day 27. The hilltop relay station was relocated on Day 30, one of four such moves that occurred throughout the trek. By this time, the robot was operating approximately $11 \mathrm{~km}$ away from the relay station, thus validating the concept of the actively pointed antennas. On day 30 , Nomad traversed $8.08 \mathrm{~km}$, taking the aggregate past two third mark to $134 \mathrm{~km}$.

On Day 31, the demonstration added another chapter to the public outreach with the first of three successful "Rover TV" shows. The next day, the robot traversed $8.2 \mathrm{~km}$, passing the $75 \%$ mark and finishing the day at $155.7 \mathrm{~km}$.

Nomad achieved its primary objective of 200 $\mathrm{km}$ traverse on Day 38, 6 days before the end of 
the mission. The final 6 days of the trek were primarily used to experimentally characterize the performance of several subsystems including navigation software, the communication system, and the locomotion system. On July 31 st, after 45 days, the satellite link was disconnected and operations ceased. Figure 18 shows daily distance subtotals, the total is $223.5 \mathrm{~km}$.

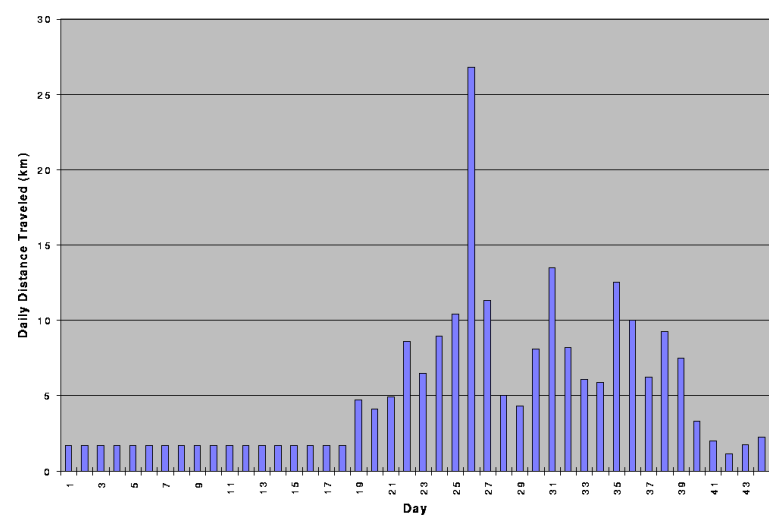

Fig. 18: Daily distance traveled by Nomad throughout the 45 days of the trek. An average daily subtotal is plotted until Day 19 when the cumulative total was $30.3 \mathrm{~km}$.

\subsection{Science experiments to learn strategies for future exploration}

The objectives for the science field experiments which occurred June 23-27 were: to provide a realistic experience for remote operators through high-quality imagery and virtual environment interfaces; to evaluate near-term planetary missions (to the Moon, Mars, and Antarctica) by training scientists, evaluating control environment appropriateness, developing and evaluating exploration strategies, and evaluating interaction in the science teams; to evaluate the importance of various imaging techniques: panospheric imaging, foveal-resolution stereo imaging, image mosaicing, and textured terrain models; and to understand the reasons for correct and incorrect scientific interpretation by collecting ground-truth and carefully evaluating scientists' methods and conclusions.

Scientists at NASA Ames Research Center conducted experiments that were simulations of remote operations on the Moon and Mars and in Antarctica.

Two Mars mission simulations provided training for site characterization and sample caching operations. The site characterization exercise, in which scientists tried to correctly characterize the climate, geology and evidence of past life, was conducted without panospheric or aerial imagery, in analog to the Mars Pathfinder mission. Scientists collaborated to analyze images from the science cameras, resulting in a slow but thorough examination. The sample caching exercise utilized all available imagery and resulted in nearly four times the area covered with a number of distinct rock types selected as samples by field geologists.

In the Lunar mission simulation, planetary scientists from NASA and the USGS attempted to perform "geology-on-the-fly", in which they assessed trafficability and surveyed gross geology while keeping the robot in motion $75 \%$ of the time. This mode of operation is appropriate for long-distance exploration or for traverse between sites of scientific interest. In a record for remote exploration, Nomad traversed 1.3 kilometers and examined 10 science sites in a single day. (Figure 19) During this test scientists also made the surprising discovery of a Jurassic fossil bed.

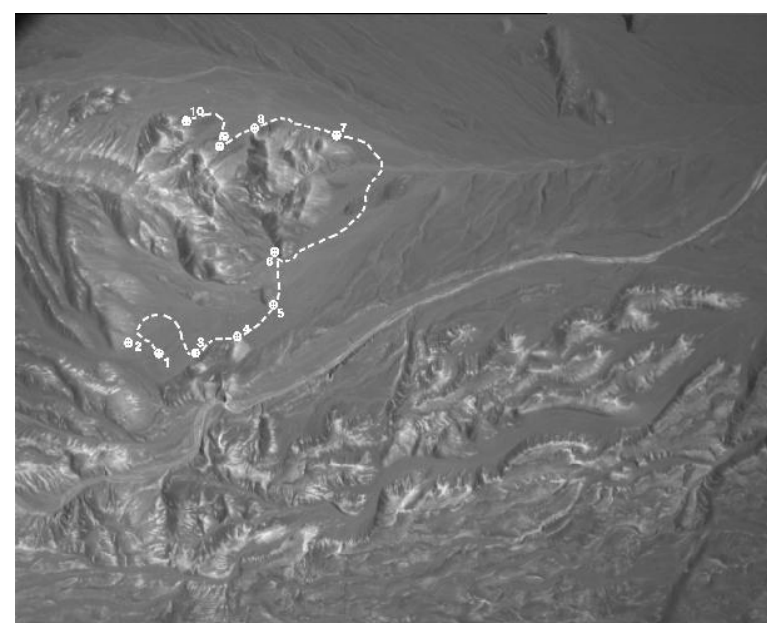

Fig. 19: Aerial image of "science-on-the-fly" site. Distance from Site 1 to 10 is $1.3 \mathrm{~km}$.

For the Antarctic test, the objective was to evaluate the feasibility of searching visually and magnetically for meteorites with a remotelycontrolled robot. On-site geologists prepared a $100 \mathrm{~m}-$ by $-5 \mathrm{~m}$ area test area with surface and buried meteorites. Nomad made a patterned search, while remote geologists looked for indicative rock features. Of three visible meteorites geologists correctly identified one meteorite (and cor- 
rectly rejected two meteorite-looking rocks). While searching with visual and magnetic sensors, they found that the readily identifiable magnetic signature helped to localize iron meteorites and significantly improved chance of discovery (three meteorites were found). [34]

Lastly, we conducted an experiment to determine the usefulness of the panospheric camera when operating with time delay. With a timedelay of 15 minutes (typical for Mars), and both with and without panoramic imagery we performed the same task: approach a science site, image sufficient features to provide an interpretation, and repeat. With the panoramic imagery, fewer uninformative science images were taken and twice as much area was examined.

While noting that the demands of remote geology require practice, geologists concurred that even without a full suite of tools for geologic exploration (like a rock hammer and a hand lens), useful and accurate analysis can be performed remotely. Although the color registration of the cameras was not precisely calibrated, color clearly reveals geologic features that are not obvious in monochrome images. Resolution is the other critical factor in remote geology. During the lunar mission simulation Nomad's high-resolution color stereo cameras revealed a previously unknown rock outcrop dating from the Jurassic era (Figure 20). Geologists identi-

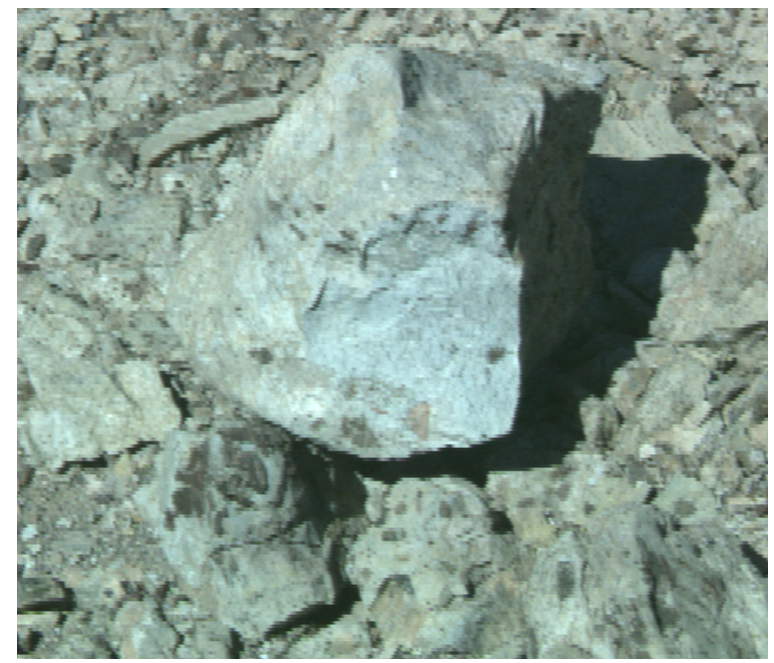

Fig. 20: Image from high-resolution cameras of Jurassic-era fossil bed (Site 4 in Figure 19).

fied the outcrop as a target of interest in an aerial image and navigated to it using panoramic imag- ery. Close inspection of several exposed rocks indicated fossilized material. Later analysis could not conclusively confirm these specific fossils but the rock outcrop was correctly characterized as a fossil bed.[37] This is the first time geologists using a robot surrogate have made such a discovery, something that was widely thought to be too difficult to do remotely. This has important implications to future planetary exploration.

\subsection{Internet-based collaborative exploration}

As part of the Atacama Desert Trek we developed a website (http://img.arc.nasa.gov/nomad) through which all data from the science experiments and much of the telemetry and imagery was available immediately.

Panospheric images, in wavelet-compressed format, were archived at $1 \mathrm{~Hz}$, and each hour one image was decompressed, dewarped and made available on the Internet. At the end of each day, the compressed images were removed from the website to make space for the next day's images. In the five days of science field experiments, 46,269 images comprising 2.8 gigabytes were archived for scientists. The entire desert trek produced more than 30 gigabytes.

The science images from the high-resolution color and wide-angle monochrome cameras were also available on the Internet. In total, 1645 science images were recorded (402 megabytes in total size) with most being high-resolution color stereo pairs for geologic analysis, and monochrome for mosaicing.

The website provided ancillary information to answer questions about the robot, its capabilities, and exact specifications of its sensors. Background information, technical information, related links, and information and photographs on the Atacama desert was included. The most popular web pages where the field logs, informative and entertaining accounts of the experience, written by one of the authors (Maimone) while in Chile.[36] We received appreciative messages from people all over the world regarding these daily accounts of the trek.

\subsection{Nomad controlled by the public}

Nomad was controlled from the Carnegie Science Center in Pittsburgh, USA, NASA Ames Research Center in Mountain View, USA and 
the Universidad Católica in Santiago, Chile. During the 250 hours of public participation, over 12,000 science center visitors were involved in the control of Nomad. In a theater audience, 32 participants were able to jointly control the direction of view projected on a $200^{\circ}$ screen. Approximately $20 \mathrm{~km}$ of Nomad's trek were under the control of audience members. The distance driven by individual novice operators was approximately $65 \mathrm{~km}$.

RoverTV was an outreach activity developed by the Centre for Metahuman Exploration at Carnegie Mellon. During three hour-long shows, Nomad's panoramic imagery was broadcast on a Pittsburgh cable television channel, and viewers were able to call in and use their telephone to control the view direction and to steer Nomad. Approximately 20 people drove Nomad in this manner, and the success of these broadcasts suggests a new approach to public interaction and robotic exploration.

The Nomad experience was significant with respect to public participation. Over 50,000 visitors to the Carnegie Science Center experienced the desert trek in some manner and more than 200 members of the public drove Nomad in the Atacama.

\section{Results, discoveries and conclusions}

The Atacama Desert Trek demonstrated capabilities necessary for robotic planetary exploration in the longest off-road robotic traverse to date. Important technologies for locomotion, high data rate communications, position estimation, safeguarded teleoperation and autonomous driving, panospheric and immersive visualization and remote geology were developed and tested. Beyond technical objectives, the Atacama Desert Trek set a new standard for operational and public outreach for robotic exploration. The outcomes of the trek are profiled in Table 2.

Nomad's in-wheel propulsion is mechanically simple and it places heavy elements like motors and gear-heads low, dropping the center of gravity and increasing stability. The averaging linkage distributes loads among all the wheels and smooths body angles and excursions, even under extreme conditions. Explicit steering eliminates the side loads, extreme power draw, and most of

\begin{tabular}{|c|c|}
\hline Topic & Outcome \\
\hline $\begin{array}{l}\text { Remote } \\
\text { Operations }\end{array}$ & $\begin{array}{l}\text { - } 201 \mathrm{~km} \text { from the science center } \\
\text { (101km by drivers, } 63 \mathrm{~km} \text { of patterns } \\
\text { and } 21 \mathrm{~km} \text { of autonomy) } \\
\text { - } 18 \mathrm{~km} \text { from NASA Ames } \\
\text { - } 2 \mathrm{~km} \text { from Santiago }\end{array}$ \\
\hline Locomotion & $\begin{array}{l}\text { - } 223.5 \mathrm{~km} \text { during the trek } \\
\text { - } 26.8 \mathrm{~km} \text { max. in a day } \\
\text { - Approx. } 100 \text { chassis open/close }\end{array}$ \\
\hline $\begin{array}{l}\text { Pano- } \\
\text { spheric } \\
\text { Camera }\end{array}$ & $\begin{array}{l}\text { - } 40,000 \text { bytes/image } \\
\text { - } 20,000-30,000 \text { images/day } \\
\text { - } 1 \text { million images at } 1 \mathrm{~Hz} \text { or better }\end{array}$ \\
\hline $\begin{array}{l}\text { Communi- } \\
\text { cation }\end{array}$ & $\begin{array}{l}\text { - } 1.5 \mathrm{Mbps} \text { mobile network } \\
\text { - Achieved } 11 \mathrm{~km} \text { separation between } \\
\text { Nomad and relay station } \\
\text { - } 16 \mathrm{hrs} / \text { day of link for } 2 \text { months }\end{array}$ \\
\hline $\begin{array}{l}\text { Safe- } \\
\text { guarded } \\
\text { Teleop }\end{array}$ & $\begin{array}{l}\text { - } 21 \mathrm{~km} \text { autonomous traverse at } 0.43 \mathrm{~m} / \\
\mathrm{s} \\
\text { - } 6 \mathrm{~km} \text { safeguarded teleops at } 0.43 \mathrm{~m} / \mathrm{s} \\
\text { - Maintained calibration for } 45 \text { days }\end{array}$ \\
\hline \begin{tabular}{|l} 
Position \\
Estimation
\end{tabular} & $\begin{array}{l}\text { - } 180-300 \mathrm{~m} \text { accuracy in } 1600 \mathrm{sq} . \mathrm{km} \\
\text { area }\end{array}$ \\
\hline Science & $\begin{array}{l}\text { - Simulated } 4 \text { planetary analog mis- } \\
\text { sions } \\
\text { - Longest robotics traverse of } 1.31 \mathrm{~km} \text { in } \\
\text { a day while performing science } \\
\text { - Detected planted meteorites using } \\
\text { cameras, a metal detector and mag- } \\
\text { netometers. }\end{array}$ \\
\hline $\begin{array}{l}\text { Public } \\
\text { Participa- } \\
\text { tion }\end{array}$ & $\begin{array}{l}\text { - Approx. 50,000 people visited Nomad } \\
\text { websites } \\
\text { - Approx. } 12,000 \text { people visited Electric } \\
\text { Horizon theatre to watch live video } \\
\text { and some drove the robot } \\
\text { - More than } 200 \text { novice drivers and sci- } \\
\text { entists drove Nomad } \\
\text { - Pittsburgh viewers drove Nomad using } \\
\text { telephones while watching imagery on } \\
\text { cable TV }\end{array}$ \\
\hline
\end{tabular}

Table 2: Atacama Desert Trek outcomes

the slippage common in skid-steered systems. The extremely wide wheelbase Nomad achieves through this transforming chassis gives it stability that belies its stowed size.

Nomad has communicated with relay stations up to $11 \mathrm{~km}$ away at data rates up to $1.5 \mathrm{Mbps}$. This is the first time this order of range and continuous data rate has been achieved from a mobile robot operating in the field. This demonstration has implication to future planetary missions where robot must travel farther from its lander or communicate directly with Earth to explore a larger areas.

Nomad operated autonomously for $10 \%$ of the trek (about $21 \mathrm{~km}$ ) using its built-in obstacle detection capability. Although it slowed down to 
avoid obstacles, it typically drove continuously at speeds up to $43 \mathrm{~cm} / \mathrm{sec}$. In addition, the safeguarded teleoperation system was tested during $7 \mathrm{~km}$ of the trek and was successful in avoiding all obstacles. The stereo cameras comprising the obstacle detection system maintained their calibration and were effectively untouched for the entire mission. Further refinement of the vehicle kinematics will enable it to operate more aggressively and to better cope with complex, obstacle strewn terrain.

Initial indications are that the ability to see all-around the robot provides drivers with a sense of position and orientation at the remote site that has been previous lacking in remote exploration, and substantially benefits and accelerates site exploration. Panoramic imagery clearly improves efficiency-it helps scientists assess the gross geology and quickly focus on key indicators. This has benefit when operating with a time delay. The combination of panoramic imagery with an intuitive graphical interface enable anyone to quickly understand how to control Nomad.

The science imaging system allowed better scientific exploration and interpretation of the remote geology than previously deployed systems. This is attributed to the threefold increase in resolution and the readily available color. The accuracy of geologic interpretations substantially improved over previous experiments with high-resolution color stereo imaging as a crucial factor.[35]

In an extended and very public experiment like the Atacama Desert Trek, the most sought after result is to survive the distance and the duration. In this regard Nomad's $223.5 \mathrm{~km}$ in 45 days, is a significant achievement.

\section{Acknowledgments}

The work presented in this paper is a collaborative effort of many people and several organizations. Nomad's success was the result of its team and the cooperation from various governmental agencies and private companies. The Nomad project was led by Red Whittaker and managed by Eric Rollins at Carnegie Mellon University where Nomad was designed and built.We thank Dimitrios Apostolopoulos, Fabio Cozman, Jesse Easudes, Alex Foessel, Carlos
Guestrin, Martial Hebert, Lalit Katragadda, Michael Krebs, Eric Krotkov, Juan León, Paul Levin, John Murphy, Paul Parker, Mike Parris, Liam Pedersen, Ben Shamah, Mark Sibenac, Jack Silberman, Reid Simmons, and Jim Teza for their tireless efforts.

NASA Ames Research Center developed the human interface and led the science experiments. The Virtual Dashboard and science package, were developed by the Intelligent Mechanisms group of NASA Ames. We thank Maria Bualat, Dan Christian, Lou Schmidt, Kurt Schwehr, Mike Sims, Hans Thomas, Deanne Tucker, and Eric Zbinden. The science mission simulations were designed and organized by Nathalie Cabrol of NASA Ames. We appreciate the efforts of the science team members: Guillermo Chong Diaz, James Dohm, George Dunfield, Virginia Gulick, Ragnhild Landheim, Pascal Lee, Rendy Keaten, Ted Roush, Carol Stoker, and Aaron Zent.

The GROK lab at the University of Iowa developed the software for display of panoramic imagery in the Telepresence Interface and supported public participation during the trek. Thanks to Steven Dow and April Rathe. At the Pontificia Universidad Católica de Chile, we thank Francisco Lira, Miguel Torres, and Andres Guesalaga for internet development and support.

Thanks are due to the Friends of Nomad [39]: Aironet, BEI, Carnegie Science Center, Center for Metahuman Exploration, Centro de Estudios Espaciales (Universidad de Chile), the Chilean Army, City of Pittsburgh, Coasin, Codelco, Dalsa, Embassy of the United States in Santiago, Entel, FINDS, Fourth Planet, Fuerza Aerea de Chile, Heinz Endowment, Human Computer Interaction Institute, Iowa Space Grant Consortium, Intel, Learning Curve Toys, LunaCorp, Mesta Electronics, Mekanismos, NASA Internet, The North Face, Pittsburgh Foundation, Pontifica Universidad Católica de Chile, RealTime Innovations, Inc., Sandia National Laboratories, Silicon Graphics, Spitz, Inc., Summus, Ltd. Trimble Navigation, Ltd., University Católica del Norte, University of Pittsburgh, and ViRtogo, Inc.

This work was supported by the NASA Space Telerobotics Program, David Lavery, Program Manager, through grants NAGW-3863 and NAGW-1175. 


\section{References}

[1] Galileo Mission at: http://nssdc.gsfc.nasa.gov/planetary/galileo.html

[2] Pathfinder Mission at: http://mars.jpl.nasa.gov/ index 1.html

[3] International Planetary Rover Efforts at: http:// www.cs.cmu.edu/ mwm/rover/

[4] D. Bapna, E. Rollins, J. Murphy, et. al., The Atacama Desert Trek: Outcomes, in: Proc. 1998 Intl. Conf. on Robotics and Automation (IEEE, Leuven, May 1998) 597-604.

[5] W. Whittaker, D. Bapna, et. al., Atacama Desert Trek: A Planetary Analog Field Experiment, in: Proc. Intl. Symp. on AI, Robotics, and Automation for Space (Tokyo, Japan, July 1997) pp. 355-360.

[6] G. Heiken, D. Vaniman, B. French, Lunar Sourcebook: A User's Guide to the Moon. (Cambridge University Press, 1991), p. 483.

[7] Marsokhod at: http://img.arc.nasa.gov/marsokhod/ marsokhod.html

[8] E. Rollins, J. Luntz, A. Foessel, et. al., Nomad: A Demonstration of the Transforming Chassis, in: Proc. 1998 Intl. Conf. on Robotics and Automation (IEEE Leuven, May 1998) 611-617.

[9] D. Apostolopoulos, Analytical Configuration of Wheeled Robotic Locomotion. Ph.D. Thesis, The Robotics Institute, Carnegie Mellon University, (Pittsburgh, 1997).

[10] M. Bekker, Theory of Land Locomotion (University of Michigan Press, Ann Arbor, 1956).

[11] D. Bapna, J. Teza, E. Rollins, W. Whittaker., An Innovative High Bandwidth Communication System for Mobile Robots, in: SAE 1997 Transactions- Journal of Aerospace, Sec. 1, Vol. 106, (SAE, 1997), 9951000 .

[12] D. Bapna, M. Martin, W. Whittaker, Earth-Moon Communication from a Moving Lunar Rover, in: Proc. of the 42nd Intl. Instrumentation Symposium, (San Diego, May 5-9, 1996) 613-622.

[13] F. Cozman, E. Krotkov, Mountain Detection and Pose Estimation for Teleoperation of Lunar Rovers, in: Proc. 1997 Intl. Conf. on Robotics and Automation (IEEE, 1997) and also at: http:// www.cs.cmu.edu/afs/cs/project/viper/www/

[14] D. Bapna, Payload Pointing from Mobile Robots. $\mathrm{Ph}$.D. Thesis, The Robotics Institute, Carnegie Mellon University, (Pittsburgh, 1998), also as: CMU-RITR-98-08.

[15] D. Bapna, E. Rollins, A. Foessel, R. Whittaker, Antenna Pointing for High Bandwidth Communications from Mobile Robots, in: Proc. 1998 Intl. Conf. on Robotics and Automation (IEEE Leuven, May 1998) 3468-3473.

[16] E. Krotkov, M. Hebert, L. Henriksen, et. al., Field Trials of a Prototype Lunar Rover under Multi-Sen- sor Safeguarded Teleoperation Control, in: Proc. Seventh ANS Topical Meeting on Robotics and Remote Systems (Augusta, 1996).

[17] E. Krotkov, M. Hebert, R. Simmons, Stereo Perception and Dead Reckoning for a Prototype Lunar Rover, in: Autonomous Robots, Vol. 2, (1995), 313331.

[18] R. Simmons, L. Henriksen, L. Chrisman, G. Whelan, Obstacle Avoidance and Safeguarding for a Lunar Rover, in: AIAA Forum on Advanced Developments in Space Robotics, (Madison, August, 1998).

[19] P. Backes, K. Tso, UMI: An interactive supervisory and shared control system for telerobotics, in: Proc. 1990 Intl. Conf. on Robotics and Automation, Vol. 2 (1990) 1096-1101.

[20] L. Conway, R. Volz, M. Walker, Tele-autonomous systems: methods and architectures for intermingling autonomous and telerobotic technology, in: Proc. of Intl. Conf. on Robotics and Automation, Vol. 2 (IEEE, 1987) 1121-1130.

[21] B. Christensen, W. Drotning, S. Thunborg, Graphical model based control of intelligent robot systems, in: Proc. of Intl. Conf. on Systems, Man, and Cybernetics, Vol. 2 (IEEE, 1991) 1069-1075.

[22] B. Hine, C. Stoker, et al., The application of telepresence and virtual reality to subsea exploration, in: Proc. AIAA, (Reno, 1996).

[23] W. Kim, Graphical operator interface for space telerobotics, in: Proc. of Intl. Conf. on Robotics and Automation, Vol. 3 (IEEE, 1993) 761-768.

[24] J. Murphy, Application of Panospheric Imaging to a Teleoperated Lunar Rover, in: Proc. of Intl. Conf. on Systems, Man and Cybernetics, Vol. 4 (IEEE, 1995).

[25] Summus Inc., at: http://www.summus.com

[26] Real-time Innovations, at: http://www.rti.com/ndds

[27] S. Bogner, D. Southwell, S. Penzes, Progress in Video Immersion using Panospheric Imaging, in: Proc. 12th Symp. on Aerospace/Defense Sensing, Simulation and Controls, Vol. 3363 (SPIE, Orlando, 1998).

[28] V. Nalwa, A true omnidirectional viewer, Technical report, Bell Laboratories, Holmdel, 1996.

[29] S. Nayar, Catadioptric omnidirectional camera, in: Proc. of Computer Vision and Pattern Recognition Conf., (IEEE, 1997) 482-488.

[30] D. Wettergreen, M. Bualat, D. Christian, et. al., Operating Nomad during the Atacama Desert Trek, in: Proc. Field and Service Robotics Conf. (Canberra, December 1997) 80-89

[31] H. Thomas, B. Hine, J. Garvey, Kilauea: A Terrestrial Analog for Studying the Use of Rovers in Planetary Science, in: Proc. Conf. of the Space Studies Institute (Princeton, May 1995).

[32] D. Christian, D. Wettergreen, M. Bualat, et. al., Field Experiments with the Ames Marsokhod Rover, in: Proc. Field and Service Robotics Conf. (Canberra, 
December 1997) 93-100.

[33] Photo-realistic Virtual Reality at: http:// img.arc.nasa.gov/StereoPipeline/topvr.html

[34] L. Pedersen, Robotic Deployment of Electro-Magnetic Sensors for Meteorite Search, in: Proc. 1998 Intl. Conf. on Robotics and Automation (IEEE, Leuven, May 1998) 618-624.

[35] N. Cabrol, G. Diaz, J. Dohm, et. al., Atacama I: Science Results of the 1997 Nomad Rover Field Test in the Atacama Desert, Chile, LPSC Report, NASA Ames Research Center, (NASA, 1998).

[36] Atacama Desert Trek Field Logs at: http:// img.arc.nasa.gov/archive/nomad/field_logs/

[37] N. Cabrol, G. Diaz, G. Dunfield, et. al., Atacama II: Nomad Rover Sample I-250697 and Implications for Fossil Characterization during Mars Exploration, LPSC Report, NASA Ames Research Center, (NASA, 1998).

[38] Atacama Desert Trek at: http://www.ri.cmu.edu/atacama-trek

[39] Friends of Nomad at: http://www.ri.cmu.edu/atacama-trek/FON/FON.html

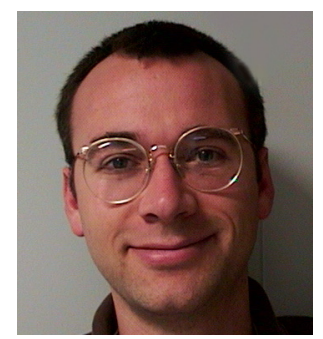

David Wettergreen is a Research Fellow at the Australian National University where he is engaged in research into control, autonomy, and learning for autonomous underwater vehicles. Previously, David was an NRC Research Associate at NASA Ames Research Center. At NASA, he developed vision-based control systems for mobile robots and participated in rover field experiments. For the Nomad project he led the NASA team that developed the human interface, communications software, science package and which ran science experiments during the trek. David completed his Ph.D. in Robotics at Carnegie Mellon University in 1995. He also holds a M.S. in Software Systems and a B.S. in Mathematics. His doctoral research concerned the generation of walking gaits for dexterous mechanisms and involved work with the Ambler, Dante I and Dante II robots. Those projects took him to Antarctica, Alaska, but most frequently to the slag heaps in Pittsburgh. His research interests include intelligence and autonomy, vision-based guidance, dexterous locomotion, and robotic exploration.

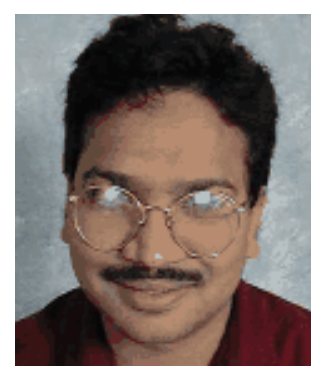

Deepak Bapna is a Division Scientist at the Precision Systems and Space Division of the BEI Sensors and Systems Co. He earned his Ph.D. in Robotics from the Robotics Institute of the Carnegie Mellon University in 1998 with a dissertation that addressed the problem of tracking from mobile robots. Deepak led the communication systems development for the 1997 Atacama Desert Trek. He received the Allen Newell Medal for Research Excellence for this work. Since March 1998, Deepak, as a Division Scientist at BEI, has been leading the new product and development group. His research interests includes robotics and automation, remote planetary exploration, control systems, robot communication, and tracking.

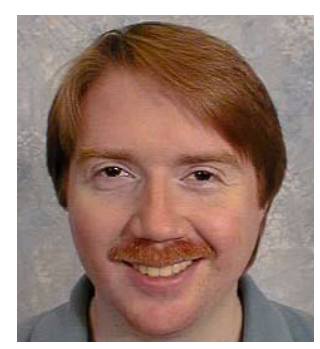

Mark Maimone is a Machine Vision researcher at NASA's Jet Propulsion Laboratory, a division of the California Institute of Technology. In 1989 he completed the International Space University's summer program, in which he participated in the design of a lunar polar orbiter. He earned his Ph.D. in Computer Science from the Computer Science Department of Carnegie Mellon University in 1996, with a dissertation that addressed problems that have plagued stereo vision systems for decades. He was then a Post-doctoral Research Associate at Carnegie Mellon's Robotics Institute, serving as Navigation and Software Lead for the 1997 Atacama Desert Trek. He received the Allen Newell Medal for Research Excellence for this work. Since December 1997, Mark has been a Machine Vision researcher at JPL working on the Long Range Science Rover, Planetary Dexterous Manipulator, and Pioneer Vision System for Chernobyl Inspection projects, delivering 3-D vision systems for autonomous robot operations and mapping. His research interests include robotic navigational autonomy, stereo vision, camera calibration, and software environments.

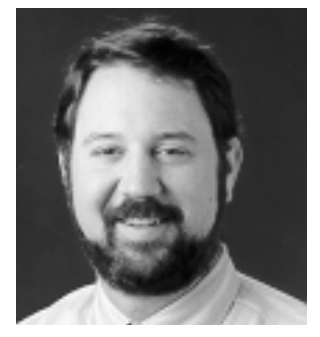

Geb Thomas is an Assistant Professor in Industrial Engineering at the University of Iowa and the director of the GROK Lab. He received his Ph.D. from The Pennsylvania State University in 1996 where he developed a gesturedriven virtual reality interface supervisory control of robots. In 1997 Geb received a National Science Foundation PostDoctoral Fellowship and worked with the Intelligent Mechanisms Group at the NASA Ames Research Center, where he developed a number of tools for the visualization of science data provided by the Marsokhod mobile robot. He is currently directing the GROK Lab's efforts in designing and building the virtual reality interface for the Pioneer robot's inspection of the Chornobyl Structure. His research interests include applications of virtual reality for mobile robotics and medicine, human computer interaction, scientific visualization and spatial perception. 